\title{
Iris Recognition Using Level Set and Local Binary Pattern
}

\author{
Brian O’Connor and Kaushik Roy
}

\begin{abstract}
This paper presents an efficient algorithm for iris recognition using the Level Set (LS) method and Local Binary Pattern (LBP). We deploy a Distance Regularized Level Set (DRLS)-based iris segmentation procedure in which the regularity of the Level Set (LS) function is intrinsically maintained during the curve propagation process. The LS evolution is derived as the gradient flow that minimizes energy functional with a distance regularization term and an external energy that drives the motion of the zero LS toward iris boundary accurately. DRLS also uses relatively large time steps in the finite difference scheme to reduce the curve propagation time. The deployed variational model is robust against poor localization and weak iris/sclera boundaries. Furthermore, we apply a Modified LBP (MLBP) in an effort to elicit the iris feature elements. The MLBP combines both the sign and magnitude features for the improvement of iris texture classification performance. The identification and verification performance of the proposed scheme is validated using the CASIA version 3 interval dataset.
\end{abstract}

Index Terms - Iris recognition, distance regularized level set, modified local binary pattern.

\section{INTRODUCTION}

Most current iris recognition algorithms perform relatively well in a strictly controlled environment. However, the iris images captured in a noisy environment produce nonideal iris images with a varying image quality and are severely affected from eyelid and eyelash occlusions, motion blurs, camera diffusions, head rotations, gaze directions, camera angles, and reflections [1]. Furthermore, the iris contours of the noisy irises are not exactly circular or elliptical and may be of any kind of shapes [2]-[6]. To mitigate the shape irregularities of the iris contours, several researchers proposed different iris segmentation methods based on active contours, including the modified Mumford-Shah segmentation model [2], Variational Level Set (VLS) [3], Fourier series expansions of the contour data [4], and Geodesic Active Contours (GAC) [5].

The segmentation approaches based on the traditional active contours may involve a huge computational time. Also, the parametric active contour-based iris segmentation scheme may be terminated due to specular reflections, the

Manuscript received October 30, 2013; revised February 17, 2014. This research was supported in part by the Army Research Laboratory (ARL) for the multi-university, Center for Advanced Studies in Identity Sciences (CASIS) and by the National Science Foundation (NSF), Science \& Technology Center: Bio/Computational Evolution in Action Consortium (BEACON).

The authors are with the Computer Science Department, North Carolina Agricultural and Technical State University, Greensboro, NC 27411 USA (e-mail: bpoconno@aggies.ncat.edu,kroy@ncat.edu). thick radial fibres in the iris and the crypts in the ciliary region. In conventional active contour method with the LS formulation, the LS function typically develops irregularities during its evolution, which may cause numerical errors and eventually destroy the stability of the evolution [7]. Addressing the above problems, we apply the Distance Regularized Level Set (DRLS) method, proposed in [7], to localize the iris contour. In the DRLS method, the regularity of the LS function is intrinsically maintained during the curve propagation. The LS evolution is derived as the gradient flow that minimizes energy functional with a distance regularization term and an external energy that drives the motion of the zero LS toward iris boundary [7]. The distance regularization term provides a unique forward-and-backward (FAB) diffusion effect, which maintains a desired shape of the LS function. Furthermore, the distance regularization eliminates expensive reinitialization and thereby avoids the induced numerical errors. DRLS also allows more efficient initialization of the LS function and uses relatively large time steps in the finite difference scheme to reduce the curve propagation time. The applied variational model is robust against poor localization and weak iris/sclera boundaries. In the multibiometric systems, proposed in [8], [9], the DRLS-based localization scheme was used to detect the iris and gait structures. A variational LS (VLS) method with a penalty term for initialization was deployed in [3] to detect the iris and pupil boundary. In this work, a more general variational LS formulation with a distance regularization term and an external energy term is applied according to the approach proposed in [7].

Elicitation of texture features from the normalized iris image plays an important role in iris recognition. Most of the state-of-the-art iris recognition algorithms utilize the local appearance descriptors such as Gabor filters, SURF, SIFT, and histograms LBP in an effort to extract the iris features. The LBP operator [10], [11] has been regarded as one of the best descriptors for the appearance of local facial regions [12] and it has been widely used in various application areas, including the face and iris recognition [13], [14]. In this paper, we propose to apply a modified LBP (MLBP), which fuses both the sign and magnitude features, to improve the feature extraction performance [11]. Though the sign component of LBP operator preserves most of the information of local difference, the magnitude component provides additional discriminant information that enhances the overall recognition accuracy.

The rest of this paper is organized as follows. Section II describes iris segmentation approach. The iris feature extraction using MLBP is discussed in Section III. Section IV reports the experimental results and Section V provides our 
conclusions.

\section{IRIS SEGMENTATION USING DRLS}

The segmentation of the nonideal iris image is a difficult task because of the noncircular shape of the pupil and the iris, and the shape differs depending on the image acquisition techniques [2]-[5]. We divide the iris segmentation process into three steps. In the first step, we apply a reflection detection process. We use simple image processing techniques and elliptical model to detect the inner (pupil) boundary of the iris in the second step. In the final step, we apply the DRLS [7] to find the exact outer boundary of the iris based on the estimated pupil boundary obtained in the previous step.

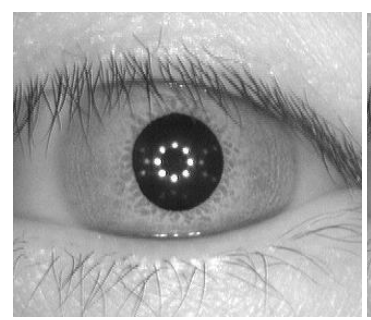

(a)

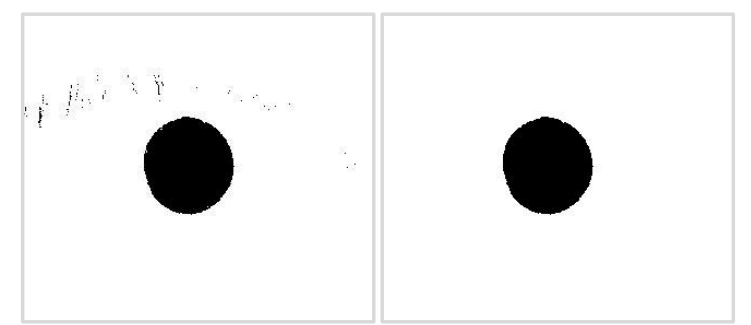

(c)

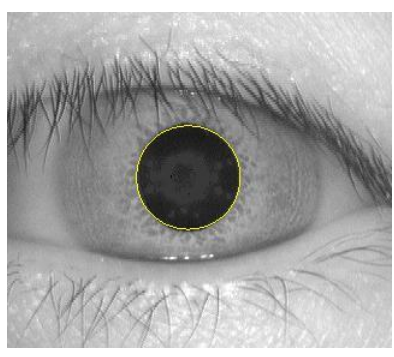

(e)

Fig. 1. (a) The original image from the CASIA version 3 interval dataset, (b) reflection elimination, (c) image after binary thresholding, (d) application of morphological closing operation, (d) isolation of pupil boundary using DLS elliptical fitting.

In the first stage of segmentation, we detect the reflection areas using a simple thresholding technique [15]. Fig. 1(b) exhibits the reflection detection result. The pupil is usually the darkest object in an image of an eye, which allows an easier detection. In the second step, we again use a simple binary thresholding to find the pupil area as shown in Fig. 1(c). After applying the morphological closing operations and obtaining the largest connected region, most of the unwanted regions are removed (see Fig. 1(d)). Prior to applying the curve evolution approach based on DRLS, we deploy Direct Least Square (DLS)-based elliptical fitting technique to isolate the pupil boundary [3], [4]. Fig. 1(e) shows the detected pupil boundary. Based on the approximation of the inner boundary, the curve is evolved by using the Level Set (LS) for accurate segmentation of the iris region. In the following paragraphs, we briefly discuss the segmentation process based on DRLS method.

In the LS formulation, let $\varphi: \Omega \rightarrow \Re$ be a LS function on a domain $\Omega$. To evolve the curve towards the iris boundary, we define the following total energy functional according to [7]:

$$
E(\varphi)=\mu R_{p}(\varphi)+\lambda L_{g}(\varphi)+v A_{g}(\varphi)
$$

where $R_{p}(\varphi)$ is the LS regularized term and $\mu>0, \lambda>0$ and $v$ are constants. The terms $L_{g}(\varphi)$ and $A_{g}(\varphi)$ in (1) are respectively defined as follows:

$$
L_{g}(\varphi) \triangleq \int_{\Omega} g \delta(\varphi)|\nabla \varphi| d x(2)
$$

and

$$
A_{g}(\varphi) \triangleq \int_{\Omega} g H(-\varphi) d x(3)
$$

where $\delta$ is the univariate Dirac function, and $H$ is the Heaviside function. The energy functional $L_{g}(\varphi)$ measures the length of the zero LS curve of $\varphi$, and $A_{g}(\varphi)$ is used to speed up the curve evolution. The LS regularized term $R_{p}(\varphi)$ can be further computed as follows:

$$
R_{p}(\varphi) \triangleq \int_{\Omega} p(|\nabla \varphi|) d x
$$

where $p$ denotes a potential function $p:[0, \infty) \rightarrow \Re$, which forces the gradient magnitude of LS function to one of its minimum points, and thus, maintains a desired shape of LS function. The LS-based curve evolution is derived as a gradient flow that minimizes the energy functional of (1). In the LS propagation, the regularity of the LS formulation is maintained by a FAB diffusion derived from the distance regularization term [7]. Therefore, the distance regularization term completely eliminates the costly reinitialization process and avoids the undesirable side effect induced by numerical errors. The DRLS is implemented with a simpler and more efficient numerical scheme than the conventional LS formulations [7]. Most importantly, relatively large time steps can be used to significantly reduce the number of iterations and computation time in the curve evolution process, while assuring sufficient numerical accuracy. In (1), $g$ denotes the edge detector function and is defined by

$$
g=\frac{1}{1+\left|\nabla G_{\sigma} * I\right|^{2}}
$$

where $G_{\sigma}$ is the Gaussian kernel with a standard deviation denoted as $\sigma$, and $I$ denotes an iris image. The convolution in (5) is used to smooth the iris image and to reduce the effect of noise. Now, the energy functional of (1) can be approximated by

$$
\begin{gathered}
E_{\epsilon}(\varphi)=\mu \int_{\Omega} p(|\nabla \varphi|) d x+\lambda \int_{\Omega} g \delta_{\epsilon}(\varphi)|\nabla \varphi| d x+ \\
v \int_{\Omega} g H_{\epsilon}(-\varphi) d x(6)
\end{gathered}
$$

where $\delta_{\epsilon}$ and $H_{\epsilon}$ denote the approximated Dirac delta function and Heaviside function, respectively. The energy functional (6) can be minimized by solving the following gradient flow 


$$
\begin{gathered}
\frac{\partial \varphi}{\partial t}=\mu \operatorname{div}\left(d_{p}(|\nabla \varphi|) \nabla \varphi\right)+\lambda \delta_{\epsilon}(\varphi) \operatorname{div}\left(g \frac{\nabla \varphi}{|\nabla \varphi|}\right)+ \\
v g \delta_{\epsilon}(\varphi)
\end{gathered}
$$

The (7) can be used to evolve the curve in an effort to detect the iris boundary using the DRLS. Fig. 2(b) shows the DRLS-based curve evolution process. Since the elicited iris regions are not exactly circular and elliptical and may be of arbitrary shapes (See Fig. 2(c)), a circle fitting strategy is applied to the extracted non-circular iris region in an attempt to mitigate the size irregularities as shown in Fig. 2(d) [3]. To convert iris region to a rectangular form, the centre values obtained through the circle fitting process and the approximated radius of such a circle are used for the unwrapping process. We unwrap the iris region to a normalized rectangular block with a fixed dimension of size $64 \times 512$ using the rubber-sheet model [4]. Fig. 2(e) shows the unwrapped image.

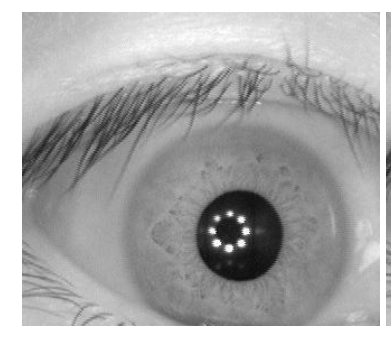

(a)

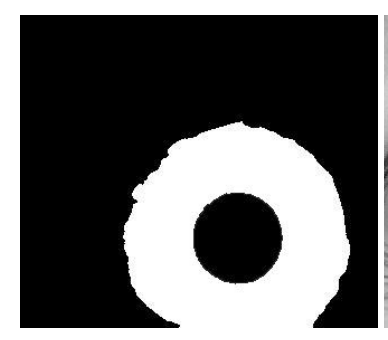

(c)

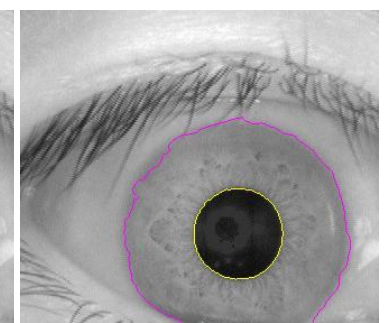

(b)

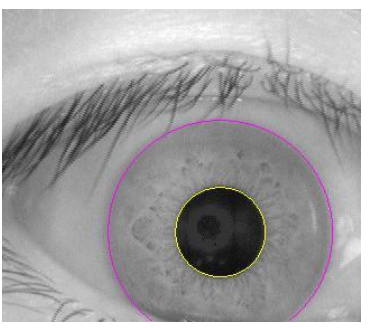

(d)

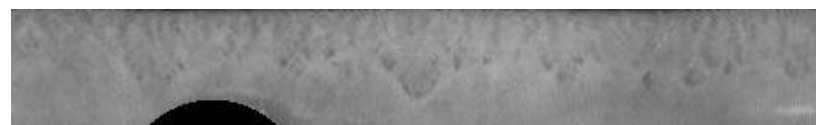

(e)

Fig. 2. (a) Original image from the CASIA version 3 interval dataset, (b) iris detection using the DRLS model, (c) binary masking of the iris with DRLS, (d) iris image after applying the circle fitting technique, and (e) normalized image.

\section{FEATURE EXTRACTION USING MLBP}

In this work, the LBP operator is used to elicit the iris features. The traditional LBP code is computed by comparing a pixel of an image with its neighboring pixels [10], [11]:

$$
L B P_{P, R}=\sum_{p=0}^{p-1} r\left(g_{p}-g_{c}\right) 2^{p}, r(x)=\left\{\begin{array}{l}
1, x \geq 0 \\
0, x<0
\end{array}\right.
$$

where $g_{c}$ denotes the gray level value of the center pixel, $g_{p}$ represents the value of the neighboring pixels of the center, $P$ represents the total number of neighboring pixels and $R$ is the radius of the neighborhood. If an image of size $I \times J$ is considered, LBP pattern is computed for each pixel of that image and a histogram is developed to represent the iris texture [11]:

$$
\begin{gathered}
\operatorname{Hist}(k)=\sum_{i=1}^{I} \sum_{j=1}^{J} f\left(L B P_{P, R}(i, j), k\right), k \in[0, K], \\
f(x, y)=\left\{\begin{array}{l}
1, \quad x=y \\
0, \text { otherwise }
\end{array}\right.
\end{gathered}
$$

where $K$ denotes the maximal LBP pattern value. Now, the local difference, $\operatorname{Dist}_{P}$, between the center pixel $g_{c}$ and the evenly spaced neighboring pixels, $g_{p}, p=0,1,2, \ldots, P-1$ is measured as Dist $_{P}=g_{p}-g_{c}$. Thus, we obtain the image local structure at $g_{c}$ with the local difference vector $\left[\right.$ Dist $_{0}, \ldots \ldots$, Dist $\left._{p-1}\right]$. Since the center intensity value, $g_{c}$ is removed, the local difference vector provides robust performance against the illumination changes. We decompose the Dist $_{P}$ into two components [11]:

$$
\operatorname{Dist}_{P}=s_{p} * m_{p} \text { and }\left\{\begin{array}{c}
s_{p}=\operatorname{sign}\left(\text { Dist }_{P}\right) \\
m_{p}=\mid \text { Dist }_{P} \mid
\end{array}\right.
$$

where $s_{p}=\left\{\begin{array}{c}1, \text { Dist }_{P} \geq 0 \\ -1, \text { Dist }_{P}<0\end{array}\right.$ and $m_{P}$ are the sign and magnitude of $\operatorname{Dist}_{P}$, respectively. In the MLBP operator, we utilize both the sign and magnitude components of Dist $_{P}$ in an effort to improve the texture extraction performance. To extract the iris texture features, first, we divide the normalized iris region into several patches and apply the MLBP on each patch (see Fig. 3). Each patch is represented by 256 sign and 256 magnitude components since we consider all the LBP patterns in this effort. We concatenate the sign and magnitude components and thus, present a single patch by $256 \times 2=512$ components.

\begin{tabular}{|l|l|l|l|}
\hline & & & \\
\hline & & & \\
\hline & & & \\
\hline & & & \\
\hline & & & \\
\hline & & & \\
\hline & & & \\
\hline
\end{tabular}

Fig. 3. Normalized image is divided into 28 sub-images.

\section{EXPERIMENTAL RESULTS AND DISCUSSIONS}

We evaluated the performance of the proposed iris recognition scheme on the CASIA version 3 interval dataset [16]. The CASIA version 3 interval iris dataset includes 2639 iris images from 249 different persons, with 396 iris classes. Most of the images were captured in two sessions with at least one month interval. The iris images are 8-bit gray level images with a resolution of $320 \times 280$. The DRLS-based segmentation approach is applied to isolate the iris boundary. Fig. 4 shows the segmentation results. We find from this figure that the proposed approach performs well for the iris images in which the iris/sclera boundaries are not clearly separable. We can also see from the Fig. 4 that the proposed approach with DRLS detects the upper and lower eyelids reasonably well. An extensive set of experiments were conducted using DRLS, and the selected parameter values were set to $\mu=0.05, v=-4.0, \lambda=5.0$, and time step, $\nabla t=3.0$. The number of iterations ranges from 50 to 300 . In this research effort, we did not include the eyelash detection technique. 
To show the effectiveness of the proposed scheme, we compared the proposed segmentation technique with the Masek's segmentation algorithm which utilizes a traditional iris localization scheme based on the Canny edge detection and Hough transform [17]. Fig. 5 shows the comparison of the proposed localization algorithm with the Masek's implementation [17]. In this effort, for comparison purpose, we only used segmentation scheme reported in [17]. It can be found from the Fig. 5 that Masek's technique fails to segment the iris images properly in all three cases (See Fig. 5(b)), while DRLS successfully localizes the iris regions for the corresponding cases (See Fig. 5(a)). As mentioned in Section III, we divided each subject's image into a multitude of different patch variations and generated MLBP feature vector for each patch. We processed every combination of patches from $1 \times 1$ to $13 \times 13$ and then compared the results of such combination of patches using the Manhattan Distance. Tables I and II report the top nine recognition accuracies for the DRLS and Masek's algorithm. It is observed from the Tables I and II that the proposed approach with DRLS provides better results than the Masek's approach for every patch configuration. The highest accuracy obtained by the DRLS is $81.45 \%$ for the $7 \times 12$ patch configuration, while the Masek's algorithm achieves the highest accuracy of $71.89 \%$ for the $5 \times 7$ patch combination.

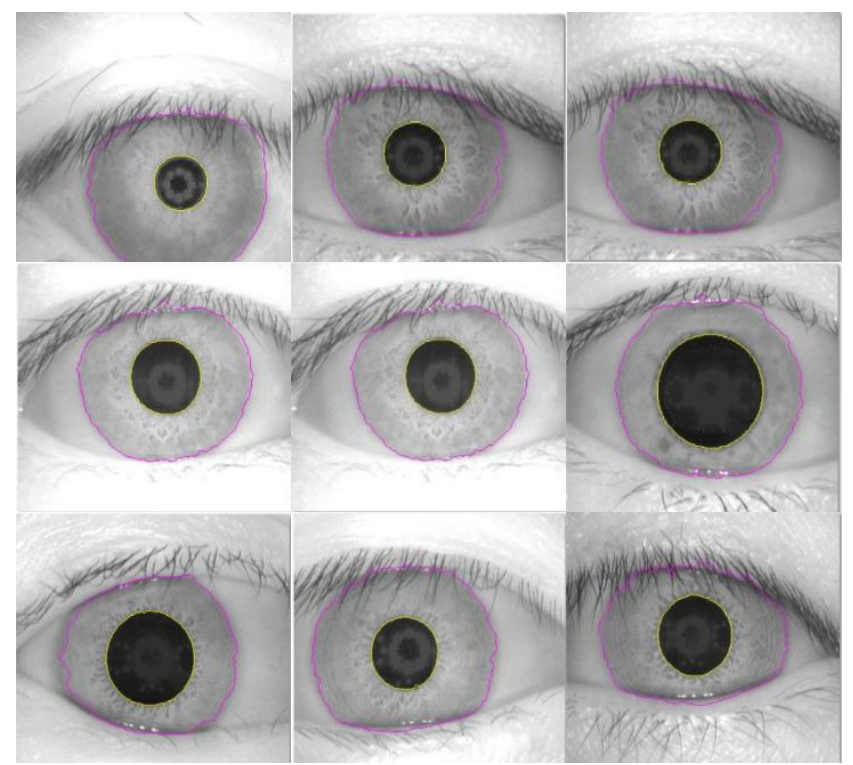

Fig. 4. Iris segmentation results on CASIA version 3 interval dataset.

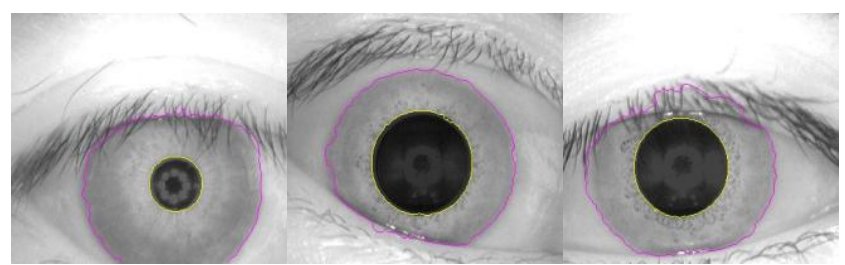

(a)

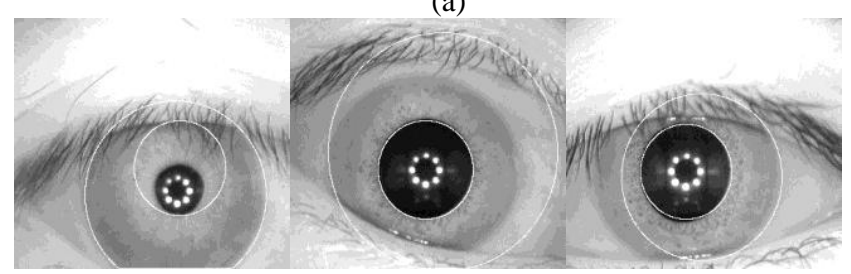

(b)

Fig. 5. Comparison between (a) DRLS and (b) Masek's [17] segmentation methods.
TABLE I: BEST MLBP PATCH COMBINATIONS (MASEK'S ALGORITHM [17])

\begin{tabular}{ll}
\hline $\begin{array}{l}\text { Patches } \\
(\text { Rows } \times \text { Columns })\end{array}$ & Accuracy \\
\hline $5 \times 7$ & 0.718987 \\
$5 \times 13$ & 0.718987 \\
$5 \times 12$ & 0.711392 \\
$5 \times 4$ & 0.708861 \\
$7 \times 12$ & 0.706329 \\
$5 \times 9$ & 0.703797 \\
$7 \times 13$ & 0.703797 \\
$5 \times 11$ & 0.698734 \\
$7 \times 4$ & 0.698734 \\
\hline
\end{tabular}

TABLE II: BEST MLBP PATCH COMBINATIONS (PROPOSED METHOD WITH DRLS)

\begin{tabular}{ll}
\hline $\begin{array}{l}\text { Patches } \\
(\text { Rows } \times \text { Columns })\end{array}$ & Accuracy \\
\hline $7 \times 12$ & 0.814516 \\
$7 \times 13$ & 0.811827 \\
$7 \times 11$ & 0.809139 \\
$5 \times 13$ & 0.798387 \\
$7 \times 10$ & 0.798387 \\
$7 \times 11$ & 0.798387 \\
$6 \times 10$ & 0.787634 \\
$6 \times 11$ & 0.784946 \\
$5 \times 11$ & 0.784946 \\
\hline
\end{tabular}

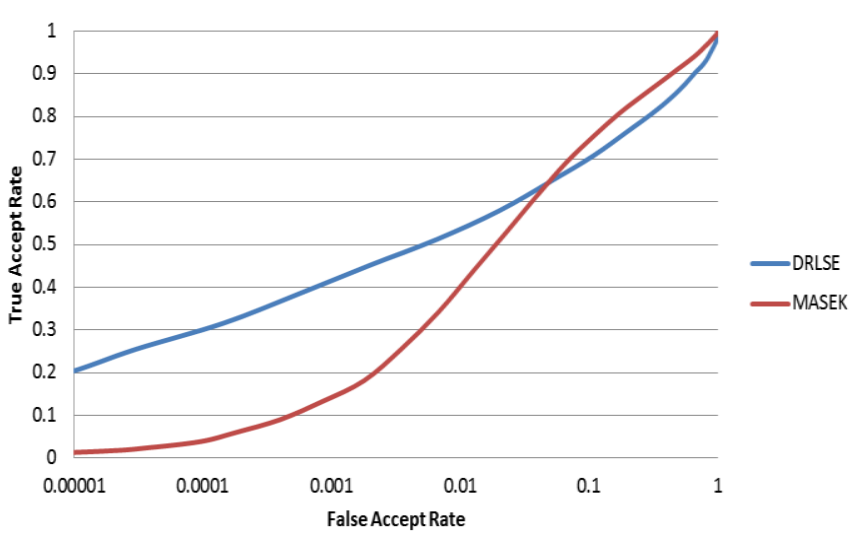

Fig. 6. ROC curve shows the comparison between DRLS and Masek's code [17].

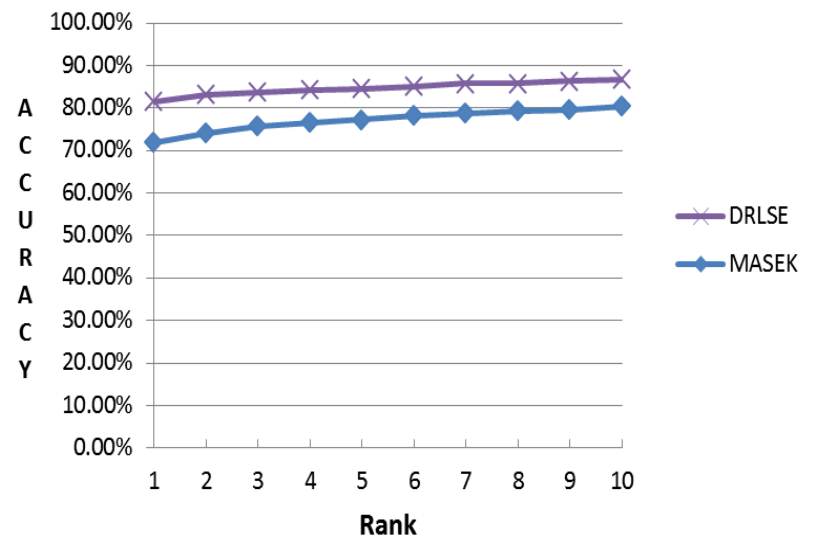

Fig. 7.Comparison of Rank (1:10) identification rates between DRLS and Masek's code [17].

Therefore, it is found that the proposed method outperforms the Masek's algorithm. In the ROC chart of Fig. 6 , we demonstrate the verification performance of the proposed scheme and also compare our method with the Masek's algorithm. We find form Fig. 6 that the proposed DRLS approach with MLBP shows better performance than the Masek's algorithm. Fig. 7 shows the rank (1 to 10) 
identification rates of the proposed and the Masek's algorithms. We observe from this figure that the proposed scheme obtains a rank-1 identification rate of $81.45 \%$, while the Masek's method receives an accuracy of $71.89 \%$. The rank-10 identification rates obtained by the proposed and Masek's methods are $88.34 \%$ and $80.50 \%$, respectively. Fig. 7 clearly shows that the active contour based approach performs relatively well than the Masek's method.

\section{CONCLUSION}

In this research effort, we have achieved two performance goals. First, we propose a new iris segmentation technique based on DRLS. This DRLS scheme is implemented with a new LS formulation and avoids the costly re-initialization process. Thus, it takes less computational time to evolve towards the iris boundary. The LS-based iris localization process outperforms the traditional Hough Transform-based segmentation approach. This method appears to have an additional benefit to simplify or eliminate the need for eyelid detection as the flexible contour fitting approach based on LS is used for iris detection. However, further research will be needed to draw a more accurate conclusion. Second, the MLBP is also applied to different patches of the unwrapped iris regions in an effort to elicit the most important iris information. The applied MLBP approach fuses the sign and magnitude features for the improvement of iris feature extraction performance. The identification and verification performance of the proposed scheme is validated on the CASIA version 3 interval dataset.

\section{ACKNOWLEDGMENT}

The authors would like to thank the ARL and the NSF for their support of this research.

\section{REFERENCES}

[1] K. Bowyer, K. Hollingsworth, and P. Flynn, "Image understanding for iris biometrics: A survey," Comp. Vis. Image Understanding, vol. 110, no. 2, pp. 281-307, 2008

[2] M. Vatsa, R. Singh, and A. Noore, "Cybernetics-Part B: Improving iris recognition performance using segmentation, quality enhancement, match score fusion, and indexing," IEEE Trans. Syst. Man and Cybernetics, vol. 38, pp. 1021-1035, July 2008.

[3] K. Roy, P. Bhattacharya, and C. Y. Suen, "Iris segmentation using variational level set method," Optics and Lasers in Engg., vol. 49, pp. 578-588, Apr. 2011.

[4] J. Daugman, "Cybernetics-Part B: New methods in iris recognition," IEEE Trans. Syst. Man and Cybernetics, vol. 37, pp. 1167-1175, Sep. 2007.

[5] S. Shah and A. Ross, "Iris segmentation using geodesic active contours," IEEE Trans. Info. Forensics and Security, vol. 4, pp. 824-836, Nov. 2009.

[6] J. Koh, V. Govindaraju, and V. Chaudhary, "A robust iris localization method using an active contour model and Hough transform," in Proc. 2010 IEEE Int. Conf. on Pattern Recog., 2010, pp. 2852-2856.

[7] C. M. Li, C. Y. Xu, C. F. Gui, and M. D. Fox, "Distance regularized level set evolution and its application to image segmentation," IEEE Trans. Image Process, vol. 19, pp. 3243-3254, Nov. 2010
[8] K. Royand and M. Kamel, "Multibiometric system using distance regularized level set method and particle swarm optimization," in Proc. 2012 Int. Conf. on Computer Vision and Graphics, 2012, pp. 590-599.

[9] K. Roy and M. Kamel, "Multibiometrics system based on level set method and particle swarm optimization," in Proc. 2012 Int. Conf. on Image Analysis and Recognition, 2012, pp. 20-29.

[10] T. Ojala, M. Pietikäinen, and T. Mäenpää, "Multire solution gray-scale and rotation invariant texture classification with local binary pattern," IEEE Trans. on Pattern Analysis and Machine Intelligence, vol. 24, pp. 971-987, Aug. 2002.

[11] Z. H. Guo, D. Zhang, and D. Zhang, "A completed modeling of local binary pattern operator for texture classification," IEEE Trans. on Image Processing, vol. 19, pp. 1657-1663, May 2010.

[12] D. Maturana, D. Mery, and A. Soto, "Face recognition with local binary patterns, spatial pyramid histograms and naive bayes nearest neighbor classification," in Proc. Int. Conf. the Chilean Comp. Science Society, 2009, pp. 125- 132.

[13] Z. N. Sun, T. N. Tan, and X. C. Qiu, "Graph matching iris image blocks with local binary pattern," in Proc. Int. Conf. Advances on Biometrics, 2005, pp. 366-372.

[14] Y. Q. He, G. Q. Feng, Y. S. Hou, L. Li, and E. Micheli-Tzanakou, "Iris feature extraction method based on LBP and chunked encoding" in Proc. 2011Int. Conf. Natural Computation, 2011, pp. 1663-1667.

[15] W. Kong and D. Zhang, "Accurate iris segmentation based on novel reflection and eyelash detection model," inProc. Int. IEEE Sympos. on Intelligent Multimedia, Video and Speech Process, 2001, pp. 263-266.

[16] CASIA-Iris Version 3 dataset. [Online]. Available: http://www.cbsr.ia.ac.cn/IrisDatabase.htm

[17] L. Masek and P. Kovesi. MATLAB source code for a biometric identification system based on iris patterns. (2003). The school of computer science and software engineering. The University of Western Australia. 2003. [Online]. Available: http://www.csse.uwa.edu.au/ pk/studentprojects/libor/sourcecode.htm

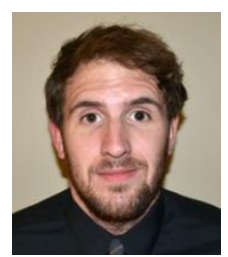

Brian O'Connor is an undergraduate student at North Carolina Agricultural and Technical State University, Greensboro, NC, USA, in the Computer Science Department. Brian is scheduled to graduate with his bachelor's degree in computer science in spring 2014.

$\mathrm{He}$ is currently working as a research assistant at North Carolina A\&T and has been in his position for a year. His research interest includes Cyber Identity, Biometrics and Machine Learning.

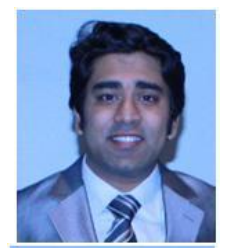

Kaushik Roy received his $\mathrm{PhD}$ from Concordia University, Montreal, QC, Canada in 2011 in computer science. He also completed his MS degree in computer science from the Concordia University in 2006 and B.Sc. degree in Computer Science from University of Rajshahi, Bangladesh in 2001.

$\mathrm{He}$ is currently an assistant professor at the Department of Computer Science, and an assistant director of the Center for Advanced Studies in Identity Sciences (CASIS), North Carolina A\&T State University, USA. Previously, he worked as a postdoctoral fellow in the Department of Electrical and Computer Engineering, University of Waterloo, ON, Canada during 2011-2012. He also taught at Rajshahi University of Engineering and Technology (RUET) as a lecturer of the Department of Computer Science and Engineering during 2001-2004.

Dr. Kaushik Roy is also the recipient of several fellowships and awards including the prestigious NSERC Visiting Fellowship, FQRNT B3 (Postdoctoral), NSERC (Doctoral) and FQRNT B2 (Doctoral). His research interests include Biometrics, cyber identity, game theory, information fusion, computer vision, machine learning, and pattern recognition. He has published 1 book, 2 book chapters, 11 journal articles and 38 conference articles. 